\title{
Leiomiosarcoma primario de mediastino que compromete el sistema de la vena cava superior. Resección quirúrgica asociada a reconstrucción venosa*
}

\author{
Drs. ROBERTO GONZÁLEZ L. ${ }^{1,2}$, RAFAEL PRATS M. ${ }^{1,2}$, CLAUDIO CIFUENTES V. ${ }^{1}$, \\ DAVID LAZO P. ${ }^{1,2}$, RAIMUNDO SANTOLAYA C. ${ }^{1,2}$, PATRICIO RODRÍGUEZ D. ${ }^{1,2}$ \\ 1 Sección de Cirugía de Tórax, Servicio Médico Quirúrgico Respiratorio, Instituto Nacional del Tórax. \\ 2 Departamento de Cirugía, Campus Oriente, Universidad de Chile. \\ Santiago, Chile.
}

\begin{abstract}
Primary leiomyosarcoma of the mediastinum involving the superior vena cava system. Surgical resection and venous reconstruction

Mediastinal tumors have multiple presentation forms and various pathological origins. Sometimes they reach a large size and can involve the system of superior vena cava by compression or infiltration. We present a 48 year old man admited for dyspnea, headache and palpitations in decubitus, for the last two months. The $\mathrm{X}$-ray and chest computed tomography showed an anterior mediastinal tumor and the tru-cut pathological study concluded leiomyosarcoma. He underwent surgical treatment; the tumor compromised the system of the superior vena cava and was resected with ligation and resection the left brachiocephalic trunk. Part of the superior vena cava was reconstructed with autologous pericardium. The right phrenic nerve was surrounded by the tumor mass and was resected in block. The patient recovered satisfactorily and was discharged on the eleventh day post surgery. The histopathologic study of the surgical specimen confirmed a leiomyosarcoma G2 $(3,100$ gr). Full adjuvant radiotherapy was done. At 15 months after surgery the patient is in good condition without evidence of recurrence or superior vena cava syndrome.
\end{abstract}

Key words: Leiomyosarcoma, mediastinum, vena cava superior, thoracic surgery, cardiovascular surgical procedures.

\section{Resumen}

Los tumores del mediastino pueden tener múltiples formas de presentación y diversos orígenes anatomopatológicos. A veces alcanzan gran tamaño y pueden comprometer por compresión o infiltración el sistema de la vena cava superior. Presentamos el caso de un hombre de 48 años que consultó por disnea, cefalea y

*Recibido el 6 de septiembre de 2010 y aceptado para publicación el 12 de enero de 2011.

No existen conflictos de interés ni apoyo financiero.

Correspondencia: Dr. Roberto González L.

José Manuel Infante 717, Santiago, Chile. Fax: 056-02-5754997

rgonzalezlagos@udec.cl 
palpitaciones en decúbito de dos meses de evolución. En la radiografía y en la tomografía computada de tórax se demostró un tumor de mediastino anterior, el estudio anatomopatológico mediante biopsia tru-cut concluyó leiomiosarcoma. Se realizó tratamiento quirúrgico, se resecó la masa tumoral que comprometía el sistema de la vena cava superior, se ligó y resecó el tronco venoso braquiocefálico izquierdo o innominado y se reconstruyó parte de la vena cava superior con parche de pericardio autólogo. El nervio frénico derecho se encontraba rodeado por la masa tumoral y se resecó junto con el tumor. El paciente evolucionó satisfactoriamente y fue dado de alta al décimo primer día post operado. El estudio de anatomía patológica de la pieza operatoria confirmó un leiomiosarcoma G2 de 3.100 gramos. Se completó tratamiento con radioterapia post operatoria. A 15 meses post operado el paciente se encuentra en buenas condiciones generales, sin evidencias de recidiva y sin síndrome de vena cava superior.

Palabras clave: Leiomiosarcoma, mediastino, vena cava superior, cirugía torácica, cirugía cardiovascular.

\section{Introducción}

Los tumores del mediastino pueden tener múltiples formas de presentación y diversos orígenes anatomopatológicos, a veces alcanzan gran tamaño y pueden comprometer por compresión o infiltración la vena cava superior (VCS) y/o sus principales vasos tributarios $^{1-6}$.

Los sarcomas corresponden a un mínimo porcentaje de los tumores de mediastino ${ }^{7,8}$. Los sarcomas de células de músculo liso (leiomiosarcomas) mediastínicos generalmente derivan de estructuras como esófago, tráquea o grandes vasos; siendo una excepción los de origen no precisado o mejor llamados leiomiosarcomas (LMS) de tejidos blandos mediastínicos ${ }^{9-14}$.

Los tumores de mediastino que infiltran la VCS se consideran en muchas oportunidades irresecables. En casos seleccionados se han descrito múltiples alternativas para reconstruir o reparar la VCS y/o sus principales vasos tributarios luego de la resección de tumores ${ }^{1-6}$.

Presentamos el caso de un paciente con un leiomiosarcoma de mediastino que comprometía el sistema de la vena cava superior y que se resecó; además se resecó el tronco venoso braquiocefálico izquierdo o innominado (TVBCI) y parte de la VCS que fue reconstruida.

\section{Caso clínico}

Hombre de 48 años, con antecedentes de tuberculosis pulmonar tratada a los 8 años de edad y hábito tabáquico suspendido hace 5 años (5 paquetes/año). Consultó por disnea progresiva (al momento de la consulta a moderados esfuerzos), cefalea y palpitaciones en decúbito de dos meses de evolución.

En la radiografía de tórax postero-anterior y lateral se demostró una lesión tumoral en el mediastino anterior (Figura 1). El estudio se complementó con tomografía computada de tórax en la que se observó un tumor de mediastino anterior que comprime y desplaza el corazón y los grandes vasos (Figuras 2 y 3$)$.

Con estos antecedentes e imágenes fue derivado a nuestro centro, evaluado por el equipo quirúrgico se decidió estudio anatomopatológico mediante biopsia

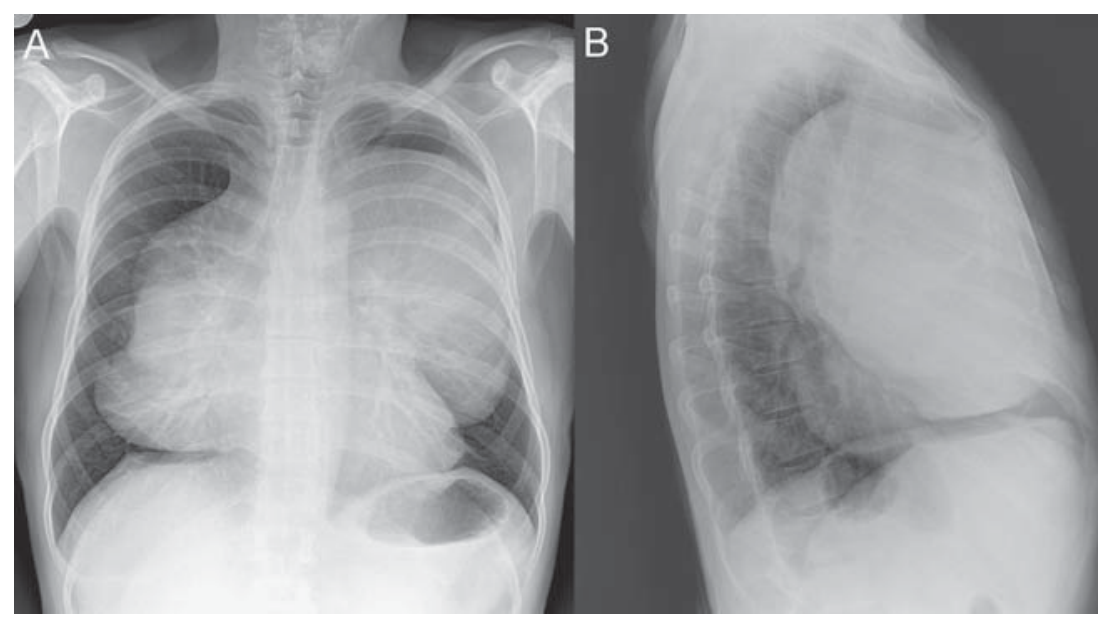

Figura 1. Radiografía de tórax postero-anterior (A) y lateral (B). 


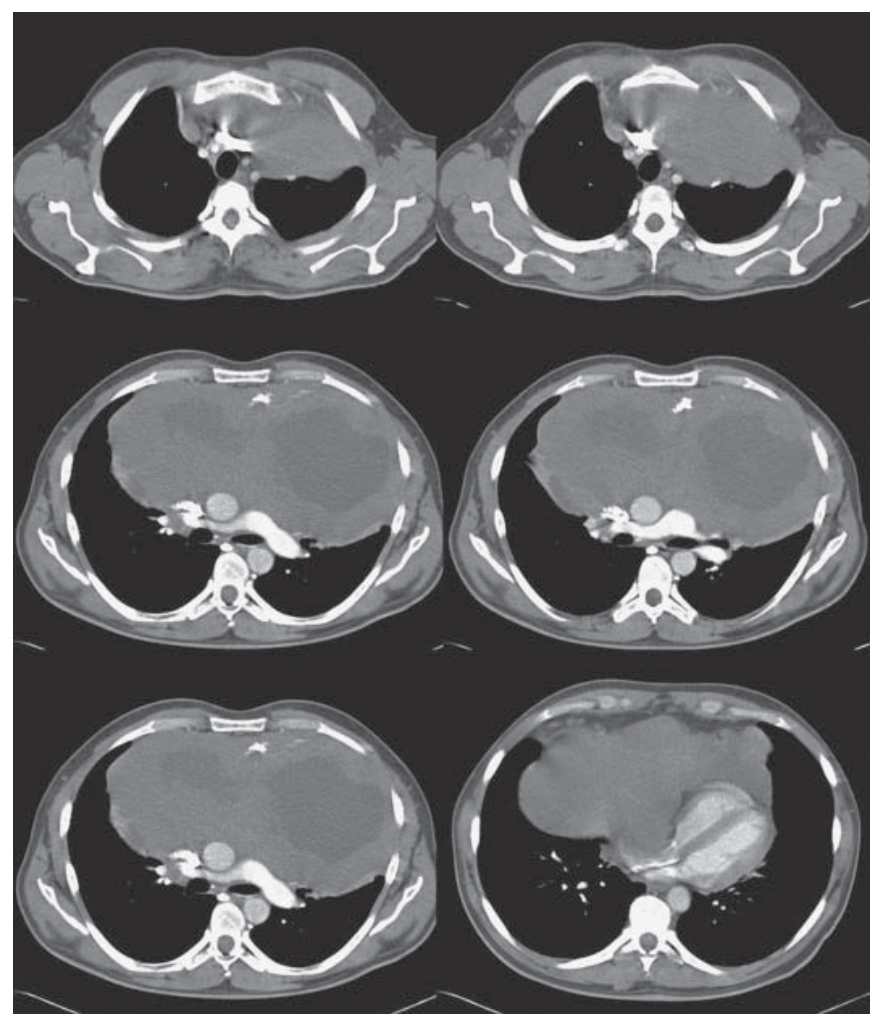

Figura 2. Tomografía computada de tórax, se observa en diversos cortes axiales la lesión tumoral y su relación con el corazón y los grandes vasos.

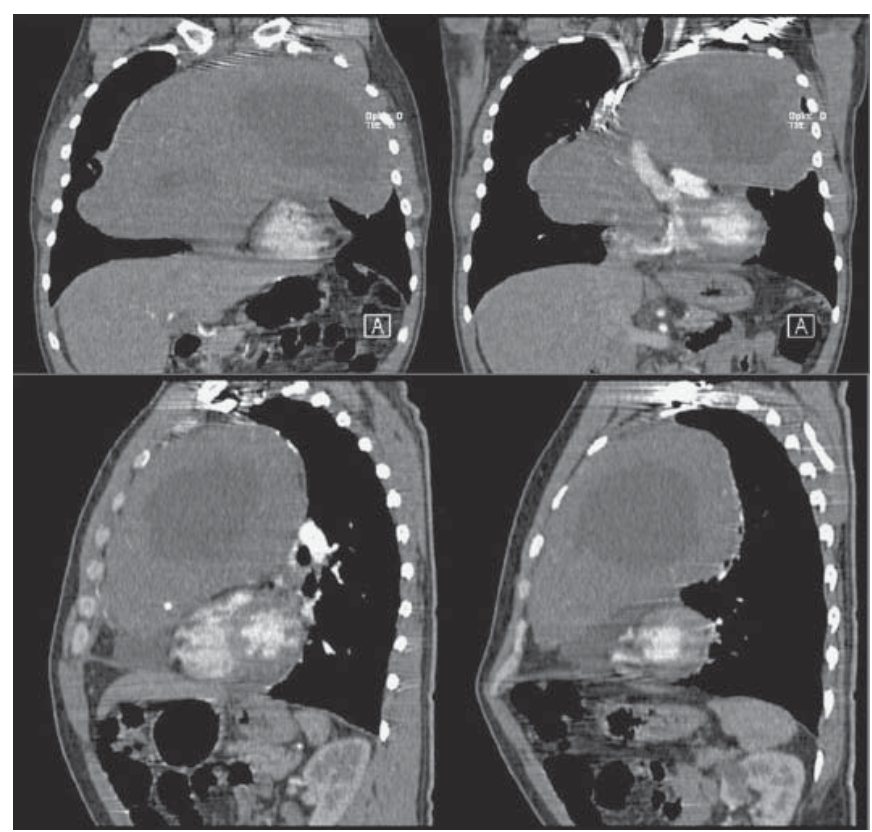

Figura 3. Tomografía computada de tórax, se observa en diversos cortes coronales y sagitales la lesión tumoral y su relación con el corazón y los grandes vasos.
Tru-cut. Con anestesia local se realizó la punción en el tercer espacio intercostal izquierdo a nivel de la línea medio clavicular. El estudio anatomopatológico contemporáneo informó que la lesión era concordante con una neoplasia de células fusadas y que la muestra de tejido era adecuada y suficiente para estudio diferido.

El estudio diferido de anatomía patológica informó que la lesión correspondía a una neoplasia de células fusadas concordantes con sarcoma de grado intermedio. Se complementó el estudio con inmunohistoquímica: el S-100 fue negativo en las células neoplásicas; el KI67 fue positivo con patrón nuclear en aproximadamente 5 a $10 \%$ de las células neoplásicas y la Actina de músculo liso fue positiva en las células neoplásicas con patrón citoplasmático fibrilar. La conclusión fue sarcoma de células fusadas con caracteres morfológicos e inmunohistoquímicos de leiomiosarcoma.

El paciente no asistió a controles e inició terapias alternativas (medidas dietéticas naturales y biomagnetismo). Luego de 5 meses consultó nuevamente en nuestro centro, reingresó con disnea a mínimos esfuerzos, baja de peso $(13 \mathrm{~kg}$ en 6 meses), Performance Status de 2 y un índice de masa corporal de 22,4. La tomografía computada de tórax no mostró cambios importantes en relación al tamaño del tumor. Se actualizó el estudio preoperatorio y se propuso cirugía.

\section{Procedimiento quirúrgico}

Se abordó con incisión tipo Clamshell (toracotomía anterior bilateral transesternal), debido al tamaño tumoral se complementó con una esternotomía media vertical inferior.

Se encontró un tumor de aspecto encapsulado, de aproximadamente $30 \times 20$ x $20 \mathrm{~cm}$, que rodeaba el TVBCI y el ángulo que éste forma con la VCS. El tumor se encontraba en contacto directo con: los vasos supra aórticos, la arteria pulmonar, el pericardio y el nervio frénico derecho que se encontraba rodeado por el tumor.

Durante la cirugía y a medida que se avanzaba en la disección, por el peso y tamaño tumoral se produjo compresión dinámica de las cavidades cardiacas, con el consiguiente deterioro de la hemodina- 
mia que se manifestó con hipotensión, taquicardia y arritmias. Uno de los integrantes del equipo quirúrgico debió sostener el tumor durante gran parte de la disección para evitar la compresión del corazón. Otro hecho ocurrido durante la disección, fue el hallazgo de un arco aórtico tipo bovino (donde el tronco arterial braquiocefálico y la arteria carótida primitiva izquierda nacen en un tronco común desde el arco aórtico).

Se resecó la masa tumoral en bloque con un fragmento de pericardio que se encontraba firmemente adherido a ésta y aparentemente infiltrado. Además, se ligó y resecó el TVBCI que se encontraba en el espesor del tumor. En la zona de la confluencia del TVBCI con la VCS, la pared de esta última se encontraba estrechamente adherida al tumor sin poder descartar infiltración. Por esto, con heparinización $(0,7 \mathrm{mg} / \mathrm{kg}$ iv) y clampaje de la VCS, se resecó un fragmento de ésta y se reconstruyó con un parche de pericardio autólogo que se suturó con punto continuo de polipropileno 6-0 (Figura 4). El tiempo de clampaje de la VCS proximal y distal fue de 5 minutos.

El nervio frénico derecho se encontraba completamente rodeado y adherido a la masa tumoral y se resecó junto con el tumor.

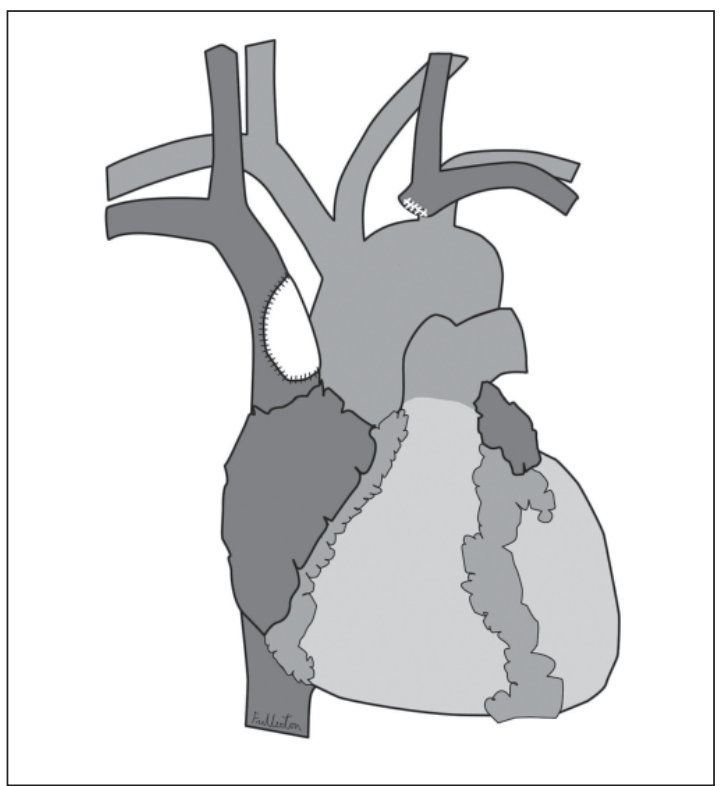

Figura 4. Esquema que muestra aspecto final de la reconstrucción venosa (el tronco venoso braquiocefálico izquierdo se resecó y la vena cava superior se reconstruyó con parche de pericardio autólogo). Se esquematiza también como hallazgo intra operatorio un arco aórtico tipo bovino.

\section{Evolución postoperatoria}

El paciente evolucionó con necesidad de ventilación mecánica no invasiva (VMNI) que se fue retirando paulatinamente hasta utilizarla sólo durante la noche. Fue dado de alta al décimo primer día post operado con VMNI nocturna domiciliaria (Figura 5).

El estudio de anatomía patológica de la pieza operatoria confirmó un leiomiosarcoma G2 de 3.100 gramos y de 30 x 21 x $19 \mathrm{~cm}$ (Figura 6). Los márgenes quirúrgicos se encontraban libres de tumor. La VCS, el TVBCI y el pericardio se encontraban firmemente adheridos pero no existía infiltración tu-

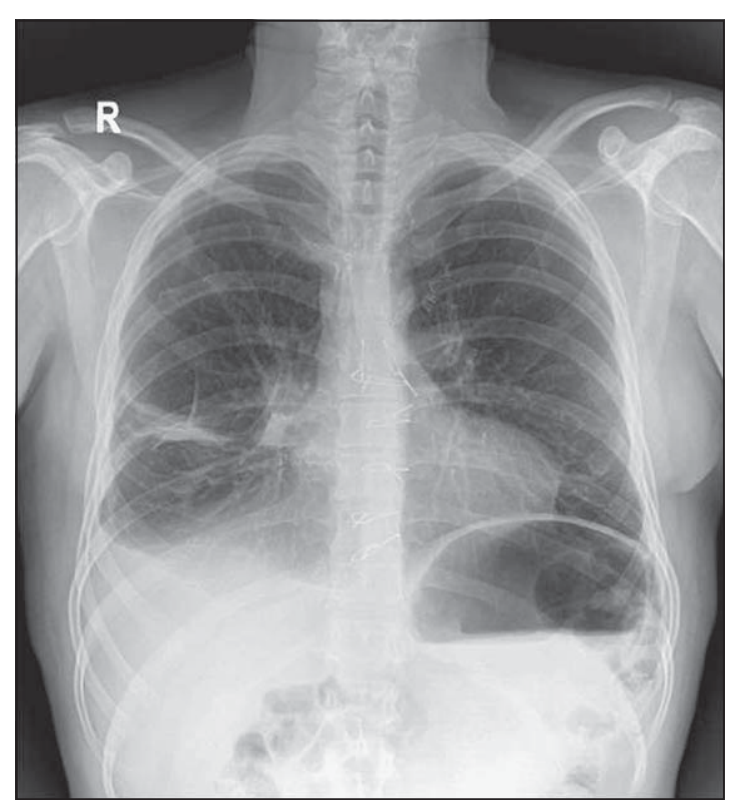

Figura 5. Radiografía de tórax postero-anterior post operatoria al momento del alta hospitalaria.

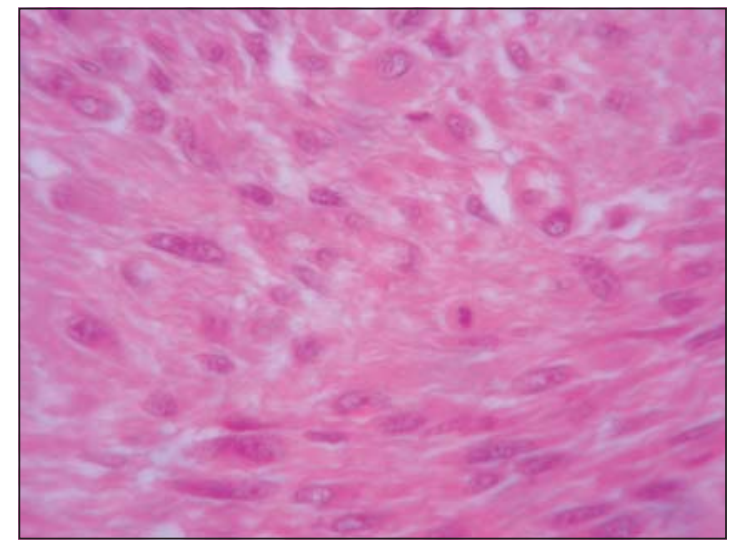

Figura 6. Anatomía patológica: Neoplasia maligna con caracteres de sarcoma de células fusadas con elementos morfológicos concordantes con leiomiosarcoma G2. 
moral. Según la clasificación GTNM para sarcomas de partes blandas, se clasificó como G2 T2bN0M0 (Etapa IIA).

Se completó tratamiento con radioterapia post operatoria, recibiendo un total de 60 Gy. Se realizó entrenamiento kinésico respiratorio y se logró el retiro definitivo de la VMNI nocturna a los 5 meses post operado. A 18 meses post operado el paciente se encuentra en buenas condiciones generales, sin síndrome de vena cava superior, con un Performance Status de 0 y sin evidencias clínicas ni radiológicas de recidiva.

\section{Discusión}

Los sarcomas de partes blandas representan menos del 1\% de los tumores malignos del adulto y aproximadamente el $2 \%$ de los tumores del mediastino $^{7,8,15}$. Los LMS corresponden entre el 5 a $10 \%$ de todos los sarcomas de partes blandas y al $9 \%$ de los sarcomas primarios del mediastino ${ }^{7,8,15,16}$.

Los LMS de tejidos blandos mediastínicos son extremadamente infrecuentes y sólo se comunican casos aislados o series pequeñas ${ }^{9-13}$. En total los casos comunicados no superan la veintena, la mayor serie corresponde a Moran y cols, que en 1994 comunicaron 10 casos $^{9}$. Resulta difícil entonces sacar conclusiones, pero en términos generales los LMS de tejidos blandos mediastínicos se presentan mayoritariamente en hombres con una presentación clínica inespecífica, con síntomas como dolor y tos, aunque pueden también ser asintomáticos. Algunos y dependiendo del tamaño, pueden presentar síntomas y signos derivados de la compresión de estructuras mediastínicas como disfagia, estridor o síndrome de vena cava superior ${ }^{9-13}$.

El origen de este tipo de tumor no es del todo claro. Las principales teorías sugieren que pueden originarse en la pared de pequeños vasos del mediastino o que sean tumores derivados de esófago, tráquea o grandes vasos que se liberan en algún momento del crecimiento $^{9-13}$. En el caso presentado, si bien es cierto el tumor se encontraba muy adherido a la VCS, al pericardio y al TVBCI (que además rodeaba), no se demostró infiltración tumoral de estas estructuras, por lo que corresponde por definición a un LMS de tejidos blandos mediastínicos o también llamado LMS primario de mediastino.

La resección quirúrgica es el tratamiento de elección en las series y casos comunicados ${ }^{9-13}$. El abordaje depende de la localización y el tamaño del tumor, en nuestro caso debido a la ubicación y al gran tamaño tumoral se abordó por una incisión tipo Clamshell, la que resultó insuficiente y se debió complementar con una esternotomía media vertical a distal. A pesar de este abordaje combinado, la di- sección y manipulación del tumor resultó compleja, especialmente por la compresión cardiaca que se produjo a medida que el tumor era disecado.

La resección completa en este tipo de tumores es un importante factor pronóstico. Según algunos autores el abordaje quirúrgico agresivo, que incluye hasta el uso de circulación extracorpórea en caso de ser necesario, sería la única alternativa real de curación ${ }^{9-13,16-18}$. La recidiva constituye uno de los principales problemas en estos tumores, en los casos que presentan recurrencias, estas ocurren frecuentemente entre los 2 a 3 años post operado ${ }^{9-13}$.

No existe consenso en relación al uso de radio o quimioterapia. Se recomienda radioterapia con el objeto de mejorar las tasas de control local en casos de resección incompleta, en márgenes quirúrgicos dudosos, mínimos o no evaluables y especialmente en tumores de alto grado de diferenciación ${ }^{7-13,16}$. En el caso presentado se decidió complementar el tratamiento con radioterapia.

El pronóstico en estos pacientes se estima según la estadificación GTNM y otros factores asociados descritos (edad, invasión vascular, necrosis, grado de resección, etc. $)^{7-13,16,17}$. Para este caso, estimamos una supervivencia de $60 \%-65 \%$ a 5 años.

La VCS y sus principales vasos tributarios pueden estar comprometidos por compresión o infiltración en tumores de mediastino o en neoplasias pulmonares $^{1-6}$.

Los beneficios a largo plazo de la resección quirúrgica extendida en cáncer pulmonar (T4) y en tumores de mediastino son motivo de controversia. En este tipo de resecciones se comunica una supervivencia a 5 años para cáncer pulmonar de $21 \%$ a $31 \%$, 4 para tumores de mediastino anterior de $45 \%$ a $53 \% 5,6$.

La factibilidad asociada a baja morbimortalidad en la resección de tumores que infiltran la VCS ha sido comunicada, pero la selección de los candidatos ideales no está del todo definida. Factores como posibilidad de resección completa, extensión del compromiso de la VCS y presencia de metástasis ganglionares deben ser considerados ${ }^{1-6,19-21}$.

En pacientes con tumores que producen síndrome de vena cava superior, la resección tumoral y la posterior reconstrucción de la VCS, produce desaparición de los síntomas y signos en la inmensa mayoría de los casos. Esta mejoría sintomática se extiende además en un alto porcentaje en el seguimiento alejado ${ }^{1-6,19-21}$.

Existen múltiples alternativas quirúrgicas para reconstruir o reparar el sistema de la VCS cuando éste se encuentra comprometido por lesiones tumorales ${ }^{1-6,18-25}$.

Si la resección es circunferencial, es decir, involucra un segmento venoso completo, se debe 
interponer un conducto o realizar un puente extra anatómico entre la VCS o uno de sus tributarios y la VCS proximal al corazón o a la aurícula derecha.

Si la resección no es circunferencial, se debe reconstruir la parte de la pared venosa resecada con algún parche protésico. En nuestro caso se realizó una resección parcial de un segmento de la VCS en el lugar de confluencia del TVBCI.

Los materiales que se pueden utilizar para remplazar un segmento de pared venosa se pueden dividir en:

Biológicos: como pericardio autólogo, vena safena autóloga, homoinjertos crio preservados, parches de pericardio bovino.

Sintéticos: como el politetrafluoroetileno o PTFE (Gore-tex $(\mathbb{R})$, tereftalato de polietileno o poliéster (Dacron $\left.{ }^{\circledR}\right)$. Estos incluso pueden ser anillados para dar un soporte externo a la pared protésica.

No existe evidencia a favor de algún tipo de material. La elección dependerá de cada caso en particular, de la disponibilidad y experiencia del equipo. Según algunos autores en caso de resecciones circunferenciales son recomendables materiales protésicos (por ejemplo PTFE anillado) y para reconstruir resecciones no circunferenciales los materiales biológicos ${ }^{20-25}$. Consideramos que la utilización de materiales biológicos y específicamente el pericardio autólogo, constituye una buena alternativa y con mejor costo beneficio en la mayoría de los casos.

Las reconstrucciones de VCS se pueden realizar en un alto porcentaje sin circulación extracorpórea $a^{1-6,19-25}$. El clampaje de la VCS puede ser parcial o total, en caso de necesitar un clampaje total, éste se puede extender hasta 45 a 60 minutos $^{1,23,24}$. Se debe asegurar un adecuado retorno venoso por la vena cava inferior. Complementariamente se pueden utilizar expansores de volumen plasmático y fármacos vasoconstrictores y si el caso lo permite se debe preservar el flujo por la vena ácigos. Con las medidas señaladas el deterioro de la hemodinámica es infrecuente durante el clampaje de la $\mathrm{VCS}^{1-6,19-25}$. Una estrategia que reduce el tiempo de clamp, es realizar en un primer tiempo la anastomosis proximal al corazón directamente en la orejuela derecha, mientras ésta se mantiene pinzada con un clamp tipo Satinsky.

Otros puntos a considerar son que este tipo de reconstrucción se debe realizar siempre con heparinización y que se deben tener presentes estructuras anatómicas como la vena ácigos, el nódulo sinusal y el nervio frénico. En el post operatorio, según el tipo de material utilizado y factores individuales de cada paciente, se recomienda en términos generales utilizar anticoagulación vía oral cuando se utilizan materiales sintéticos y antiagregación plaquetaria en caso de materiales biológicos ${ }^{1-6,19-25}$.
Como conclusiones de este caso podemos decir que, los leiomiosarconas primarios del mediastino son tumores muy infrecuentes, pueden alcanzar un gran tamaño, comprometer estructuras como la VCS y que su tratamiento es la resección quirúrgica. En algunos casos la resección extendida de tumores mediastínicos que comprometen la VCS es recomendada. Existen varias alternativas y consideraciones para reconstruir la VCS y/o sus principales vasos tributarios.

\section{Agradecimientos}

Al Dr. Demian Fullerton M., por la confección de la figura quirúrgica.

\section{Referencias}

1. Lanuti M, De Delva PE, Gaissert HA, Wright CD, Wain JC, Allan JS, et al. Review of superior vena cava resection in the management of benign disease and pulmonary or mediastinal malignancies. Ann Thorac Surg. 2009;88:392-7.

2. Park BJ, Bacchetta M, Bains MS, Downey RJ, Flores R, Rusch VW, et al. Surgical management of thoracic malignancies invading the heart or great vessels. Ann Thorac Surg. 2004; 78:1024-30.

3. Shintani Y, Ohta M, Minami M, Shiono H, Hirabayashi $\mathrm{H}$, Inoue $\mathrm{M}$, et al. Long-term graft patency after replacement of the brachiocephalic veins combined with resection of mediastinal tumors. J Thorac Cardiovasc Surg. 2005;129:809-12.

4. Dartevelle PG, Chapelier AR, Pastorino U, Corbi P, Lenot B, Cerrina J, et al. Long-term follow-up after prosthetic replacement of the superior vena cava combined with resection of mediastinal-pulmonary malignant tumors. J Thorac Cardiovasc Surg. 1991;102:259-65.

5. Spaggiari L, Leo F, Veronesi G, Solli P, Galetta D, Tatani B, et al. Superior vena cava resection for lung and mediastinal malignancies: a single-center experience with 70 cases. Ann Thorac Surg. 2007;83:223-9.

6. Chen KN, Xu SF, Gu ZD, Zhang WM, Pan H, Su WZ, et al. Surgical treatment of complex malignant anterior mediastinal tumors invading the superior vena cava. World J Surg. 2006;30:162-70.

7. Burt M, Ihde JK, Hajdu SI, Smith JW, Bains MS, Downey R, et al. Primary sarcomas of the mediastinum: results of therapy. J Thorac Cardiovasc Surg. 1998;115:671-80.

8. Paquette M, Truong PT, Hart J, Jones SO, Martens B, Christie JL, et al. Primary sarcoma of the mediastinum: a report of 16 cases referred to the British Columbia Cancer Agency. J Thorac Oncol. 2010;5:898-906.

9. Moran CA, Suster S, Perino G, Kaneko M, Koss MN. 
Malignant smooth muscle tumors presenting as mediastinal soft tissue masses. A clinicopathologic study of 10 cases. Cancer 1994;74:2251-60.

10. Conner WC, Fink GW, McGinnis KM, Alfieris GM. Surgical management of leiomyosarcoma of the mediastinum. Ann Thorac Surg. 2004;77:334-6.

11. Hirano H, Kizaki T, Sashikata T, Maeda T, Yoshii Y. Leiomyosarcoma arising from soft tissue tumor of the mediastinum. Med Electron Microsc. 2003;36:52-8.

12. Eroğlu A, Kürkçüoğlu C, Karaoğlanoğlu N, Gürsan N. Primary leiomyosarcoma of the anterior mediastinum. Eur J Cardiothorac Surg. 2002;21:943-5.

13. D'Aiuto M, Veronesi G, Pompilio G, Gasparri R, Presicci F, Galetta D, et al. Extended right pneumonectomy with partial left atrial resection for primary leiomyosarcoma of the mediastinum. J Thorac Cardiovasc Surg. 2005;129:694-5.

14. Catena F, Bianchi R, Ansaloni L, Pinna AD. Hemothorax caused by rupture of a primitive thoracic leiomyosarcoma of the thoracic aorta: description of a case and literature review. J Thorac Cardiovasc Surg. 2008;135:688-9.

15. Daugaard S. Current soft-tissue sarcoma classifications. Eur J Cancer 2004;40:543-8.

16. Svarvar C, Böhling T, Berlin O, Gustafson P, Follerås $\mathrm{G}$, Bjerkehagen B, et al. Clinical course of nonvisceral soft tissue leiomyosarcoma in 225 patients from the Scandinavian Sarcoma Group. Cancer 2007;109:282-91.

17. Gustafson P, Willén H, Baldetorp B, Fernö M, Akerman M, Rydholm A. Soft tissue leiomyosarcoma. A population-based epidemiologic and prognostic study of 48 patients, including cellular DNA content. Cancer 1992;70:114-9.
18. Vaporciyan AA, Rice D, Correa AM, Walsh G, Putnam $\mathrm{JB}$, Swisher S, et al. Resection of advanced thoracic malignancies requiring cardiopulmonary bypass. Eur J Cardiothorac Surg. 2002;22:47-52.

19. Suzuki K, Asamura H, Watanabe S, Tsuchiya R. Combined resection of superior vena cava for lung carcinoma: prognostic significance of patterns of superior vena cava invasion. Ann Thorac Surg. 2004;78:1184-9.

20. Picquet J, Blin V, Dussaussoy C, Jousset Y, Papon $\mathrm{X}$, Enon B. Surgical reconstruction of the superior vena cava system: indications and results. Surgery 2009; 145:93-9.

21. Spaggiari L, Thomas P, Magdeleinat P, Kondo H, Rollet G, Regnard JF, et al. Superior vena cava resection with prosthetic replacement for non-small cell lung cancer: long-term results of a multicentric study. Eur J Cardiothorac Surg. 2002;21:1080-6.

22. Politi L, Crisci C, Montinaro F, Andreani M, Podzemny V, Borzellino G. Prosthetic replacement and tangential resection of the superior vena cava in chest tumors. J Cardiovasc Surg. (Torino) 2007;48:363-8.

23. García A, Flores RM. Surgical management of tumors invading the superior vena cava. Ann Thorac Surg. 2008;85:2144-6.

24. Sekine Y, Suzuki H, Saitoh Y, Wada H, Yoshida S. Prosthetic reconstruction of the superior vena cava for malignant disease: surgical techniques and outcomes. Ann Thorac Surg. 2010;90:223-8.

25. Okereke IC, Kesler KA, Rieger KM, Birdas TJ, Mi $\mathrm{D}$, Turrentine MW, et al. Results of superior vena cava reconstruction with externally stented-polytetrafluoroethylene vascular prostheses. Ann Thorac Surg. 2010;90:383-7. 